\title{
Vagus Nerve Stimulation for Treatment-Resistant Depression: Behavioral and Neural Effects on Encoding Negative Material
}

\author{
Hugo D. Critchley, DPhil, MrCPsych, Penelope A. Lewis, DPhil, Michael Orth, MD, PhD, Oliver Josephs, PhD, \\ Ralf Deichmann, PhD, Michael R. Trimble, FRCPsych, and Raymond J. Dolan, FmedSci
}

\begin{abstract}
Objectives: Vagus nerve stimulation (VNS) can improve depression. Cognitive models of depression highlight an over-representation of negative thoughts and memories, with depressed individuals showing memory facilitation for negative material. We hypothesized that the antidepressant action of VNS may emerge through corrective influences on 'negativity bias' in memory. We therefore examined the impact of VNS on emotional memory and its underlying brain activity. Methods: We tested a single patient undergoing VNS for treatment-resistant depression (TRD). Stimulation was set at a 30/66-second on/off cycle during three encoding blocks when the patient viewed randomly presented positive, negative, and neutral words. Following each block, VNS was switched off and the patient identified previously seen words from distractors in a subsequent recognition memory task. The patient was scanned using functional magnetic resonance imaging (fMRI) during the first encoding block. Results: There was robust recall of negative material viewed during 'off' cycles of VNS but subsequent memory of negative words was attenuated during active VNS ('on' cycles). VNS did not influence memory for neutral and positive words. With neuroimaging, direct modulatory effects of VNS were observed in dorsomedial, dorsolateral, and orbital regions of the prefrontal cortex. Moreover, during encoding of negative words, compared with positive and neutral words, VNS also modulated activity within orbitofrontal, ventromedial and polar prefrontal cortices, midcingulate cortex, and brain stem. Conclusions: Our observations show that VNS can interfere with memory of negative information, an effect that may contribute to its antidepressant role. Neuroimaging implicated regions including the ventral and medial prefrontal cortex as an underlying neural substrate.
\end{abstract}

$\mathbf{f M R I}=$ functional magnetic resonance imaging; SAR $=$ Specific Absorption Ratio; SPM = statistical parametric mapping; $\mathbf{T R D}=$ treatment-resistant depression; VNS = vagus nerve stimulation.

\section{INTRODUCTION}

$\mathrm{O}$ bservations of epilepsy patients show that Vagus Nerve Stimulation (VNS) with the VNS Therapy System (Cyberonics Inc., Houston, TX) can affect mood, suggesting VNS as a potential therapy for severe depression (1-4). Correspondingly, VNS gained FDA approval in 2005 for the adjunctive long-term treatment of chronic or recurrent treatment-resistant depression for patients 18 years of age or older who are experiencing a major depressive episode and have not had an adequate response to four or more adequate antidepressant treatments (5).

Stimulation of the human vagus nerve activates both afferent and efferent axons. An estimated $80 \%$ of vagus nerve fibers are afferent, conveying information about internal visceral state. Animal models show VNS influences thalamocortical excitability, similar to low-level challenges such as the baroreflex (6). Neuroimaging studies in epileptic and depressed patients confirm that VNS alters activity within thalamus and cortex, including ventromedial prefrontal and orbitofrontal cortices $(7,8,9)$. These regions are implicated in mood regulation, where functional and

From the Wellcome Department of Imaging Neuroscience (H.D.C., P.A.L., O.J., R.D., R.J.D.) and Raymond Way Unit (M.O., M.R.T.), Institute of Neurology, University College London, Queen Square, London.

Address correspondence and reprint requests to Hugo D. Critchley, CISC, Brighton and Sussex Medical School, University of Sussex, Brighton, BN1 9PX, UK. E-mail: H.Critchley@bsms.ac.uk

Received for publication March 21, 2006; revision received August 4, 2006.

DOI: 10.1097/PSY.0b013e31802e106d structural abnormalities manifest in patients with severe idiopathic depression (10).

Influential cognitive models emphasize a negativity bias in the formation of depressive schemata that maintain chronic mood disorder. Specifically, negative cognitions and memories form the target of cognitive behavioral treatments for depression $(11,12)$. A reliable finding is that negative material is remembered more strongly by depressed patients compared with nondepressed controls $(13,14,15)$. Even in healthy controls, negative events are preferentially recalled in negative moods (16). Notably, antidepressants alter the balance between memory for positive versus negative material (17). VNS is reported to enhance recognition memory in patients with epilepsy (18), leading to the prediction that selective modulation of emotional memory may contribute to mechanisms by which VNS may alleviate depressive symptoms.

\section{METHODS}

We tested a 48-year-old male patient who was implanted with a VNS device for treatment-resistant depression (TRD) seven months earlier. At the time of study, he took no medication (including antidepressants). Clinical scales showed partial symptom improvement since initiation of VNS (Hamilton Rating Scale of Depression score: 34 before treatment; 25 at study exit). Consistent with the timecourse of VNS treatment effects, depressive symptoms continued to improve over ensuing months (Hamilton score after 12 months was 18). The patient gave full informed consent for our study, which was approved by the local Ethics Committee.

The patient performed three 'encoding' blocks, interleaved with three corresponding 'test' blocks, of a recognition memory task. In the encoding blocks, he made semantic (abstract self-referential) judgments (16) while viewing randomized presentations of positive, negative, and neutral words from a standard series (ANEW: Affective Norms for English Words (19)). Normative (population) ratings of the valence of these words were available (19) and negative and positive words were selected from $1 / 3$ extremes of this 
A

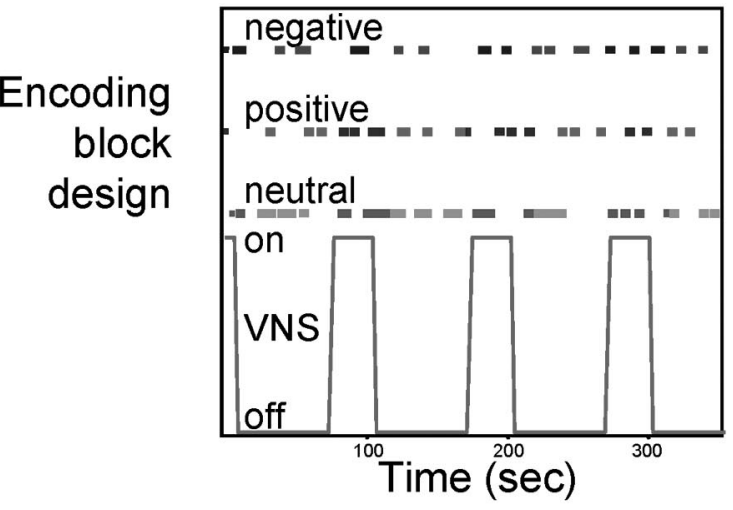

C
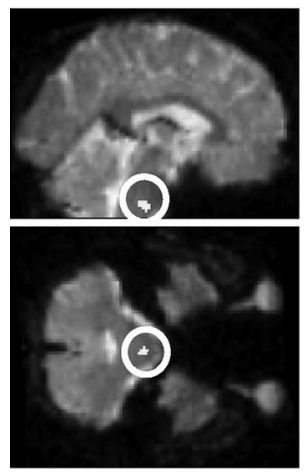

Brainstem
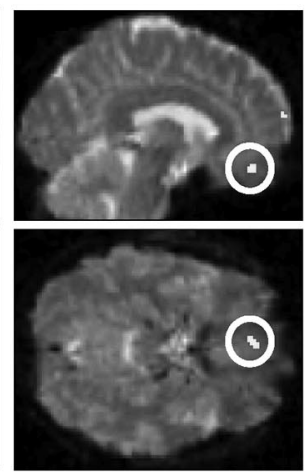

ventromedial pfc
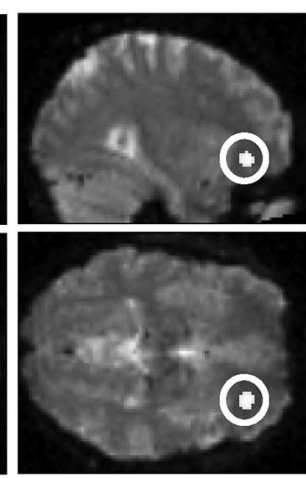

area 47
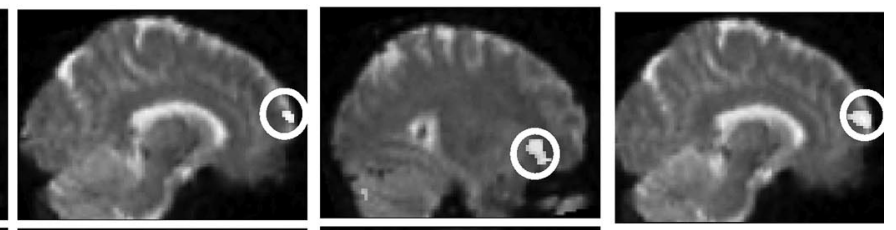

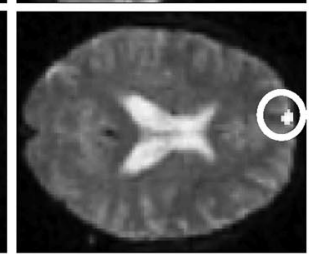

frontal pole

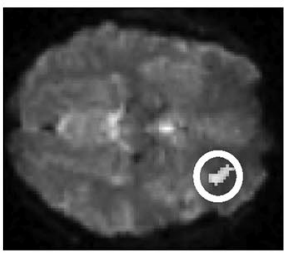

area 47

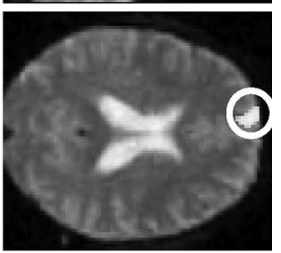

frontal pole

\section{Selective effects of VNS on encoding of negative words (interaction)}

\section{Conjunction with activity for items subsequently forgotten}

Figure 1. (A) The panel illustrates the encoding block design. Vagus nerve stimulation (VNS) was set to a 30 second 'on' / 66 second 'off' cycle as the patient viewed randomized 1 second presentations of positive, negative, and neutral words (representing discrete non-overlapping events). During the first of three encoding blocks, the patient was scanned using fMRI, signaling the onset of the VNS before scanning by a button press. (B) Across recognition memory blocks performed 20 minutes after each of the three encoding blocks, the patient made significantly more recognition errors to words encoded when the stimulator was active (i.e., in the 30 second 'on' period). This effect was almost entirely attributable to interference with the encoding of negative words (which, compared with normative data were relatively enhanced when the stimulator was 'off'). The bar chart depicts the patient's recognition memory performance for each word category across all recognition blocks (filled = stimulator 'on' at encoding). (C) Activity in a discrete set of brain regions reflected the selective behavioral impact of VNS on the encoding of negative words. VNS enhanced the activity brain stem (pons) ventromedial prefrontal cortex, right anterior insula/lateral orbitofrontal (area 47) cortex, and frontal pole during the processing of negative (but not positive or neutral) words that were subsequently recognized. Interestingly, activity within these regions, particularly area 47 and frontal pole, also predicted subsequent forgetting of words during the first encoding task, illustrated in a conjunction analysis depicted in 2 panels on the right. Significant activity relating to this interaction is plotted on a normalized T2*weighted echoplanar volume from the patient to illustrate fully the field of view. Data are presented at $p<.001$, uncorrected for clusters of 10 or more contiguous voxels. Peak coordinates and magnitudes of the effects are given in Table 1.

valence range. In each encoding block, 88 words (44 neutral, 22 negative, and 22 positive) were presented with 32 null events at a stimulus onset asynchrony (SOA) of 3 seconds. VNS $(20 \mathrm{~Hz}, 500 \mu \mathrm{s}, 2.25 \mathrm{mAmp})$ was set at a 30 seconds-'on' and 66 seconds-'off' cycle during each encoding block (changed from a duty cycle of 30 seconds-'on', 3 minutes-'off') (Figure 1A). To synchronize timing of VNS with presentation of stimuli, the patient signaled before the start of each encoding block. Each encoding block began with the onset of a tickling throat sensation (nonpainful) that indicated the beginning of a 30 second 'on' period of active VNS. Twenty minutes after each of the encoding blocks, with VNS turned off, the patient performed a corresponding recognition memory block, in which he was required to distinguish the previously presented 88 'old' words from 133 'new' words (67 neutral, 33 positive, and 33 negative for each session; 1 second presentation, SOA 3 seconds; 2 choice response for recognition). Stimuli and timing parameters for encoding and recognition tasks were identical to those used in an earlier study of healthy subjects (16).

For technical reasons, neuroimaging data were acquired using fMRI only over the period of the first encoding block. The patient was removed from the scanner after the first encoding block to turn off the stimulator and perform the corresponding recognition block. Difficulties occurred with scanning another subject during this interval (specifically high SAR (Specific Absorption Ratio) readings), attributed to a relative incompatibility of the (nonstandard) transmit-receive coil with the scanner (see below). These difficulties were not resolved that day, so the study was continued for the subsequent two encoding blocks (and all three recognition blocks) with the patient outside the scanner.

The fMRI was conducted using a Siemens Sonata (1.5T) scanner fitted with a transmit-receive head coil (replacing a standard body-transmit setup) for necessary safety and technical constraints of scanning patients with implanted VNS. During the first encoding block, $350 \mathrm{~T} 2 *$-weighted EPI volumes were acquired (44 slices/volume, $2 \mathrm{~mm}$ slice thickness, $1 \mathrm{~mm}$ interslice gap, bandwidth $2298 \mathrm{~Hz} /$ pixel, matrix $64 \times 64$, FoV $192 \mathrm{~mm}$, $\mathrm{TR} / \mathrm{TE}=3960 / 50 \mathrm{~ms}$, isotropic spatial resolution $3 \mathrm{~mm}, 90^{\circ}$ flip angle, $30^{\circ}$ tilt of the image slice from axial toward coronal orientation to avoid signal dropouts in the orbitofrontal cortex (20)). This functional dataset was corrected for slice-timing, realigned with movement correction, transformed into standard space, spatially smoothed (FWHM $12 \mathrm{~mm}$ ), and regressed against the experimental design matrix using SPM2 (21). Presentations of positive, negative, and neutral words during VNS 'on' and VNS 'off' conditions during 
TABLE 1. Changes in Brain Activity during Vagus Nerve Stimulation and Word Encoding

\begin{tabular}{|c|c|c|c|c|}
\hline Location & Side & Size & Coordinates & $\mathrm{T}$ \\
\hline \multicolumn{5}{|l|}{ Main effect of VNS stimulation (increases) } \\
\hline Dorsolateral prefrontal cortex & $\mathrm{L}$ & 15 & $-56,22,20$ & 3.63 \\
\hline Dorsolateral prefrontal cortex & $\mathrm{L}$ & 12 & $-42,20,38$ & 3.46 \\
\hline Medial orbitofrontal cortex & $\mathrm{L}$ & 10 & $-18,24,-24$ & 3.38 \\
\hline \multicolumn{5}{|l|}{ Main effect of VNS stimulation (decreases) } \\
\hline Superior lingual gyrus & $\mathrm{R}$ & 56 & $18,42,-8$ & 4.30 \\
\hline Dorsomedial prefrontal cortex & - & 114 & $4,28,50$ & 4.17 \\
\hline Angular gyrus & $\mathrm{L}$ & 47 & $-16,-70,56$ & 4.05 \\
\hline Orbitofrontal cortex & $\mathrm{R}$ & 35 & $22,44,-20$ & 3.88 \\
\hline Superior temporal sulcus & $\mathrm{L}$ & 122 & $-50,-30,-2$ & 3.88 \\
\hline Intra-parietal sulcus & $\mathrm{R}$ & 56 & $46,-44,60$ & 3.81 \\
\hline Cerebellar vermis & - & 53 & $2,-58,-20$ & 3.61 \\
\hline \multicolumn{5}{|l|}{ Main effect of encoding words } \\
\hline Intra-parietal sulcus & $\mathrm{L}$ & 624 & $-32,-60,52$ & $6.52^{a}$ \\
\hline Sensorimotor cortex & $\mathrm{R}$ & 525 & $42,-20,58$ & $5.90^{\circ}$ \\
\hline Sensorimotor cortex & $\mathrm{L}$ & 620 & $-42,-26,60$ & $5.83^{a}$ \\
\hline Occipital cortex & $\mathrm{L}$ & 276 & $-4,-86,-6$ & $4.75^{a}$ \\
\hline Occipital cortex & $\mathrm{R}$ & 195 & $10,-84,4$ & $4.46^{a}$ \\
\hline Dorsolateral prefrontal cortex & $\mathrm{R}$ & 313 & $46,30,22$ & $4.72^{a}$ \\
\hline Dorsolateral prefrontal cortex & $\mathrm{L}$ & 192 & $-52,10,36$ & $4.34^{a}$ \\
\hline Supplementary motor area & - & 236 & $0,0,54$ & $4.46^{a}$ \\
\hline \multicolumn{5}{|c|}{ Main effect of processing negative words (increases, vs. positive and neutral words) } \\
\hline Insula & $\mathrm{L}$ & 231 & $-54,14,0$ & $5.25^{a}$ \\
\hline Lingual gyrus & $\mathrm{R}$ & 310 & $12,-84,2$ & 4.43 \\
\hline Frontal pole & - & 104 & $-8,62,18$ & 4.51 \\
\hline Anterior insula & $\mathrm{R}$ & 172 & $36,24,-4$ & 4.22 \\
\hline Dorsolateral prefrontal cortex & $\mathrm{L}$ & 81 & $-36,44,22$ & 4.06 \\
\hline \multicolumn{5}{|c|}{ VNS effects on activity during encoding of neutral words (enhancement) } \\
\hline Intra-parietal sulcus & $\mathrm{R}$ & 187 & $36,-44,60$ & 4.53 \\
\hline Intra-parietal sulcus & $\mathrm{L}$ & 25 & $-20,-34,60$ & 3.57 \\
\hline Orbitofrontal cortex & $\mathrm{R}$ & 14 & $30,32,-26$ & 3.61 \\
\hline Insula & $\mathrm{L}$ & 15 & $-36,6,6$ & 3.47 \\
\hline \multicolumn{5}{|c|}{ VNS effects on activity during encoding of neutral words (attenuation) } \\
\hline Mid cingulate & - & 16 & $8,0,50$ & 3.20 \\
\hline Mid cingulate & - & 10 & $-10,-2,44$ & 3.19 \\
\hline \multicolumn{5}{|c|}{ Selective effect of VNS on processing of negative words (VNS by valence interaction) } \\
\hline \multicolumn{5}{|c|}{ More activity to negative when stimulator on } \\
\hline Orbitofrontal cortex & $\mathrm{R}$ & 83 & $30,38,-8$ & 4.09 \\
\hline Frontal pole & $\mathrm{L}$ & 36 & $-10,66,18$ & 4.04 \\
\hline Ventral pons & - & 27 & $2,-24,-44$ & 3.69 \\
\hline Ventromedial prefrontal cortex & - & 23 & $-6,44,-16$ & 3.57 \\
\hline Insula & $\mathrm{R}$ & 11 & $62,14,-4$ & 3.41 \\
\hline \multicolumn{5}{|l|}{ Less activity to negative when stimulator on } \\
\hline Mid cingulate gyrus & $\mathrm{L}$ & 71 & $-6,-18,34$ & 3.74 \\
\hline Occipitoparietal cortex & $\mathrm{L}$ & 19 & $-18,-84,42$ & 3.66 \\
\hline Superior parietal lobule & $\mathrm{R}$ & 14 & $46,-40,60$ & 3.50 \\
\hline
\end{tabular}

Activity surviving voxel-wise significance of $p<.001$, uncorrected and extent threshold of 10 contiguous voxels.

${ }^{a}$ Activity also surviving false discovery rate (FDR) correction for multiple comparisons ( $p<.05$, corrected).

the first encoding block were modeled as events with a canonical hemodynamic response. Subsequently forgotten words were modeled separately. Additionally, a boxcar regressor modeled each VNS 'on' and 'off' period. Our analyses focused on activity for words of all valances that were subsequently recognized to optimize the number of exemplars and exclude incidental confounds. Nevertheless, we also performed a conjunction analysis to demonstrate commonalities in regional activation reflecting the selective VNS effects on negative information processing at encoding (i.e., interaction: negative versus other words with stimulator on versus off) and activity that occurred when words were subsequently forgotten. The conjunction analysis tested for significant regional activity present in both conditions $(21,22)$, in this case validating the inference that the activity within some regions is specifically modulated by VNS during processing of negative words and also predictive of subsequent forgetting.

Significant neural activity is reported at a voxel-wise threshold of $p<.001$, uncorrected (extent threshold 10 contiguous voxels) (Table 1). Where activity also surpasses a false discovery rate (FDR) correction for multiple comparisons at $p<.05$, this is indicated in Table 1 to highlight the most robust activation effects.

\section{RESULTS}

Healthy adults typically show superior memory for positive, relative to negative, words in recognition memory tasks. Thus, a parallel study in healthy controls (16), using identical 
stimuli and similar methods to the present study, reported recognition accuracy at $50 \%$ to $55 \%$ for positive words, compared with $35 \%$ to $45 \%$ for negative words.

In this study, behavioral data indicate that the patient showed a potentiated recognition memory for negative words encoded during 'off' periods of stimulation across all three encoding blocks, when over $50 \%$ of negative words were subsequently recognized with confidence. However, active VNS during encoding significantly modulated the patient's subsequent memory for negative words: compared with off periods, stimulation 'on' periods were associated with selective impairment of the encoding of negative words $\left(\chi^{2}=9.55\right.$, $p<.01$ ) (Figure 1B). Although we used the standard metric for recognition accuracy (hits-false alarms), it is important to note this effect was driven by changes in the encoding of negative words, because there were very few false alarm trials (across recognition sessions, $8 \%$ of new positive words, $8 \%$ of new negative words, and $4 \%$ of new neutral words were misattributed as 'old'; in the first recall (scanned) session, rates were 4/33 for negative, $3 / 33$ new positive, and 4/66 new neutral).

Evidence of efferent effects of VNS on visceral response was limited to subjective throat-tickling at the onset of 'on'periods and a trend for heart rate to decrease during VNS (mean on: 82.2 beats per minute versus off: 82.7 beats per minute, $p=.09$ ).

Analysis of brain activity during the first encoding block revealed that the 30-second 'on' periods of VNS induced enhancement of activity within dorsolateral prefrontal cortex and medial orbitofrontal cortex and attenuated activity within parietal lobe, occipitotemporal visual cortices, dorsomedial prefrontal, right lateral orbitofrontal cortices, and cerebellar vermis (Table 1). Analyses of word encoding focused in an event-related manner on those words that were subsequently recognized: as a main effect, encoding of words enhanced activity within regions implicated in visual attention and reading, including occipital, dorsolateral prefrontal, and bilateral parietal cortices. Somatomotor cortex activation, relating to the response judgment made to each event of the encoding task, was also observed in this context. Compared with positive and neutral words, negatively-valenced words selectively activated right anterior insula, a region implicated as an interface between internal arousal states and feeling states $(23,24)$. During the encoding of neutral words, VNS enhanced activity within parietal, insula and orbitofrontal cortex, and attenuated mid cingulate responses.

In addition to these main effects (and the interaction of VNS on neutral word encoding), we tested directly for changes in regional neural activity that reflected the observed behavioral impact of VNS on the specific encoding of negative words (i.e., VNS (on versus off) $\times$ word valence (negative versus positive + neutral)). This interaction was associated with a selective enhancement of frontal polar, ventromedial prefrontal, brain stem, and right anterior insula/orbitofrontal (including area 47) activity (Figure 1C). There was also a decrease in midcingulate activity when negative words were processed during VNS. This interaction used only trials that were subsequently remembered. Nevertheless, enhanced activity within the same regions also predicted the subsequent forgetting of words in this first encoding block. This conjunction effect was most marked for activity in frontal pole and right area 47 , as formally demonstrated in a conjunction analysis (Figure 1C).

\section{DISCUSSION}

This single case report represents a 'proof of principle', highlighting one potential behavioral mechanism by which VNS may therapeutically influence depression via an action on emotional memory. Moreover the neuroimaging component of the study identifies in a single patient, putative neural substrates through which negativity biases in cognitive processing (here during emotional encoding) may be acutely influenced by VNS. In contrast to an observation in epilepsy patients (18), we observed no general facilitation of memory during the 'on' phase of VNS in our single patient. Instead, the patient, who showed strong recognition memory of negative words encoded with the stimulator off, demonstrated a selective disruption of encoding of negatively-valenced material during active VNS, an effect with a putative neural substrate localized to regions including medial and ventral prefrontal cortices.

However our observations are limited to an acute study of a single depressed patient; hence we cannot argue a definitive mode of therapeutic action of VNS in treatment-resistant depression. Moreover, previous neuroimaging studies of VNS in depression have examined acute effects and not related them to treatment outcome $(8,9)$. Nevertheless, our findings cohere with evidence emphasizing the role of cognitive and mnemonic influences on maintenance of depression $(13,14,15,17)$ and a theoretical framework underlying cognitive treatments for depression $(11,12)$. With these necessary qualifications, our study suggests a plausible behavioral and neurobiological mechanism associated with a physical treatment of depression. The neuroimaging component of the study showed that VNS directly modulated activity in orbitofrontal cortex (implicated in affective representations and learning (25)) and dorsal prefrontal cortex (associated with memory encoding and attention (26)). Moreover we show a neural correlate to the selective effect of VNS on the encoding of negative words: activity was enhanced within brain stem and right orbitofrontal, polar, and ventromedial prefrontal cortices, whereas activity in midcingulate was attenuated. The ventral and medial prefrontal cortical regions are perhaps the most significant in understanding these emotion-related effects in the context of depression. Representations of visceral arousal, relaying in brain stem, may be ultimately expressed in right insula/orbitofrontal cortex as subjective emotional feeling states $(23,24)$ and contribute to emotional control processes ascribed to area 47 (27). The ventromedial prefrontal cortex is typically activated by positive stimuli (28) and deactivated by high attentional demand and increased sympathetic tone (29). Activity in both these regions is related to mood congruence effects in healthy controls at encoding and retrieval (16). Significantly, abnormal activity within 
adjacent subgenual and orbitofrontal regions is a central neurobiological marker for depression (10).

One potential interpretation of our imaging data are that VNS augments reward-related activity within ventromedial prefrontal cortex, simultaneously interfering with central expression of emotional arousal within insula and more lateral orbitofrontal cortex. Nevertheless no behavioral augmentation of memory for positive stimuli was observed (perhaps because performance may have been close to ceiling), and the effect was an interference with (normalization of) enhanced encoding and recollection of negative word. Speculatively, the predictable afferent perturbation caused by VNS may overshadow and compete with representations (derived from peripheral feedback or central efference-copy) of visceral stress responses engendered by negative words. During encoding, associative mismatch (16) of visceral response to valenced stimuli may selectively impair subsequent memory for emotionally-arousing negative material.

Complementary brain mechanisms are implicated in memory enhancement for emotional material. Hippocampus-dependent episodic and declarative memories for emotional material are enhanced by co-activation of the amygdala $(30,31)$, perhaps more so when there is an associative learning of context $(32,33)$. Where amygdala activity is observed at encoding of emotional word items in subsequent recognition memory tasks, it is enhanced during encoding of both positive and negative items (34). This absence of valence-specificity suggests that amygdala activity may be insufficient to account for negativity bias at encoding in depressed patients. We did not observe amygdala engagement during encoding of emotional words in the present study, perhaps because of low power (small number of forgotten items in the first encoding session). Nevertheless, our neuroimaging findings point to a central role of ventral and medial polar prefrontal cortical region in mediating the memory effect of VNS on the encoding of emotionally negative material. This finding is paralleled by studies of mood congruent memory in controls (16) and processing of emotional stimuli in depressed patients (35).

Our single case report highlights a plausible mnemonic mechanism though which VNS may influence (acutely) clinical features of a treatment-resistant depression. However, it remains unknown if the normalization of emotional memory by VNS, correcting a negativity bias, contributes to the evolution of a therapeutic effect of VNS over time $(12,15)$. The patient, though slightly improved after seven months of VNS, remained significantly depressed at the time of testing but continued to improve further over ensuing months. Our study presented a rare opportunity to examine whole brain responses to vagus nerve stimulation in a nonepileptic patient. This is the first neuroimaging study to examine the neural substrate of interactions between VNS with a cognitive or emotional process, and illustrates that robust neurophysiological data may now be obtained using fMRI to enable single subject analyses.

This work was supported by a Wellcome Senior Fellowship in Clinical Science to H.D.C. and a Wellcome Trust Programme Grant to R.J.D. We are very grateful for the participation of our volunteer.

\section{REFERENCES}

1. Handforth A, DeGiorgio CM, Schachter SC, Uthman BM, Naritoku DK, Tecoma ES, Henry TR, Collins SD, Vaughn BV, Gilmartin RC, Labar DR, Morris GL 3rd, Salinsky MC, Osorio I, Ristanovic RK, Labiner DM, Jones JC, Murphy JV, Ney GC, Wheless JW. Vagus nerve stimulation therapy for partial-onset seizures: a randomized active-control trial. Neurology 1998;51:48-55.

2. George MS, Sackeim HA, Marangell LB, Husain MM, Nahas Z, Ballenger JC, Rush AJ. Vagus nerve stimulation. A potential therapy for resistant depression? Psychiatr Clin North Am 2000;23:757-83.

3. Sackheim HA, Rush AJ, George MS, Marangell LB, Husain MM, Nahas Z, Johnson CR, Seidman S, Giller C, Haines S, Simpson RK Jr, Goodman RR. Vagus nerve stimulation (VNS) for treatment-resistant depression: efficacy, side effects, and predictors of outcome. Neuropsychopharmacology 2001;25:713-28.

4. Marangell LB, Rush AJ, George MS, Sackeim HA, Johnson CR, Husain MM, Nahas Z, Lisanby SH. Vagus nerve stimulation (VNS) for major depressive episodes: one year outcomes. Biol Psychiatry 2002;51:280-7.

5. Office of Device Evaluation. VNS Therapy System p970003s050. Available at: http://www.fda.gov/cdrh/pdf/p970003s050.html. Accessed December 18, 2006.

6. Henderson LA, Richard CA, Macey PM, Runquist ML, Yu PL, Galons JP, Harper RM. Functional magnetic resonance signal changes in neural structures to baroreceptor reflex activation. J Appl Physiol 2004;96:693-703.

7. Chae JH, Nahas Z, Lomarev M, Denslow S, Lorberbaum JP, Bohning DE, George MS. A review of functional neuroimaging studies of vagus nerve stimulation (VNS). J Psychiatr Res 2003;37:443-55.

8. Lomarev M, Denslow S, Nahas Z, Chae JH, George MS, Bohning DE. Vagus nerve stimulation (VNS) synchronized BOLD fMRI suggests that VNS in depressed adults has frequency/dose dependent effects. J Psychiatr Res 2002;36:219-27.

9. Mu Q, Bohning DE, Nahas Z, Walker J, Anderson B, Johnson KA, et al. Acute vagus nerve stimulation using different pulse widths produces varying brain effects. Biol Psychiatry 2004;55:816-25.

10. Drevets WC, Price JL, Simpson JR Jr, Todd RD, Reich T, Vannier M, Raichle ME. Subgenual prefrontal cortex abnormalities in mood disorders. Nature 1997;386:824-7.

11. Beck AT. Cognitive therapy and the emotional disorders. New York: Meridian; 1976

12. Teasdale JD. Negative thinking in depression: cause, effect or reciprocal relationship? Adv Behav Res Ther 1983;5:3-25.

13. Lloyd GG, Lishman WA. Effect of depression on the speed of recall of pleasant and unpleasant experiences. Psychol Med 1975;5:173-80.

14. Clark DM, Teasdale JD. Diurnal variation in clinical depression and accessibility of memories of positive and negative experiences. $\mathrm{J}$ Abnormal Psychol 1982;91:87-95.

15. Watkins PC, Vache K, Verney SP, Muller S, Matthews A. Unconscious mood-congruent memory bias in depression. J Abnormal Psychol 1996; 105:34-41.

16. Lewis PA, Critchley HD, Smith AP, Dolan RJ. Brain mechanisms for mood congruent memory facilitation. Neuroimage 2005;25:1214-23.

17. Harmer CJ, Shelley NC, Cowen PJ, Goodwin GM. Increased positive versus negative affective perception and memory in healthy volunteers following selective serotonin and norepinephrine reuptake inhibition. Am J Psychiatry 2004;161:1256-63.

18. Clark KB, Naritoku DK, Smith DC, Browning RA, Jensen RA. Enhanced recognition memory following vagus nerve stimulation in human subjects. Nat Neurosci 1999;2:94-8.

19. Bradley MM, Cuthbert BN, Lang PJ. Affective Norms for English Words (ANEW). Technical Manual and Affective Ratings. Gainesville, FL: The Center for Research in Psychophysiology, University of Florida. 1998.

20. Deichmann R, Gottfried JA, Hutton C, Turner R. Optimised EPI for fMRI studies of the orbitofrontal cortex. Neuroimage 2003;19:430-41.

21. Wellcome Department of Imaging Neuroscience. Statistical Parametric Mapping. Available at: http://www.fil.ion.ucl.ac.uk/spm. Accessed December 18, 2006.

22. Price CJ, Friston KJ. Cognitive conjunction: a new approach to brain activation experiments. Neuroimage 1997;5:261-70.

23. Critchley HD, Wiens S, Rotshtein P, Ohman A, Dolan RJ. Neural systems supporting interoceptive awareness. Nat Neurosci 2004;7:189-95.

24. Craig AD. Interoception: the sense of the physiological condition of the body. Curr Opin Neurobiol 2003;13:500-5.

25. Rolls ET. The brain and emotion. Oxford: OUP. 1999. 
26. Nyberg L, Cabeza R, Tulving E. PET studies of encoding and retrieval: the HERA model. Psychonom Bull Rev 1996;3:135-148.

27. Eisenberger NI, Lieberman MD. Why rejection hurts: a common neural alarm system for physical and social pain. Trends Cogn Sci 2004;8:294-300.

28. O’Doherty J, Critchley H, Deichmann R, Dolan RJ. Dissociating valence of outcome from behavioral control in human orbital and ventral prefrontal cortices. J Neurosci 2003;23:7931-9.

29. Nagai Y, Critchley HD, Featherstone E, Trimble MR, Dolan RJ. Activity in ventromedial prefrontal cortex covaries with sympathetic skin conductance level: a physiological account of a "default mode" of brain function. Neuroimage 2004;22:243-51.

30. Phelps EA. Human emotion and memory: interactions of the amygdala and hippocampal complex. Curr Opin Neurobiol 2004;14:198-202.

31. Strange BA, Otten LJ, Josephs O, Rugg MD, Dolan RJ. Dissociable human perirhinal, hippocampal and parahippocampal roles during verbal encoding. J Neurosci 2002;22:523-8.

32. Maratos EJ, Dolan RJ, Morris JS, Henson RN, Rugg MD. Neural activity associated with episodic memory for emotional context. Neuropsychologia 2001;39:910-20.

33. Smith AP, Stephan KE, Rugg MD, Dolan RJ. Task and content modulate amygdala-hippocampal connectivity in emotional retrieval. Neuron 2006; 49:631-8.

34. Kensinger EA, Schacter DL. Amygdala activity is associated with the successful encoding of item, but not source, information for positive and negative stimuli. J Neurosci 2006;26:2564-70.

35. Elliott R, Rubinsztein JS, Sahakian BJ, Dolan RJ. The neural basis of mood-congruent processing biases in depression. Arch Gen Psychiatry 2002;59:597-604. 